\title{
Ex vivo Rezafungin Adsorption and Clearance During Continuous Renal Replacement Therapy
}

\author{
Soo Min Jang ${ }^{\text {a }}$ Grayson Hough ${ }^{b}$ Bruce A. Mueller \\ a Department of Pharmacy Practice, Loma Linda University School of Pharmacy, Loma Linda, CA, USA; \\ ${ }^{b}$ Cidara Therapeutics, San Diego, CA, USA; ' Department of Clinical Pharmacy, University of Michigan \\ College of Pharmacy, Ann Arbor, MI, USA
}

\section{Keywords}

Rezafungin · Drug clearance · Continuous venovenous hemofiltration · Ex vivo

\section{Abstract \\ Background/Aims: To determine adsorption and trans- membrane clearances $\left(\mathrm{CL}_{\mathrm{TM}}\right)$ of rezafungin, a novel long-act- ing echinocandin, in continuous venovenous hemofiltration (CVVH). Methods: A validated ex vivo bovine blood CVVH model using polysulfone and AN69 hemodiafilters was used to evaluate urea and rezafungin $\mathrm{CL}_{\mathrm{TM}}$ at 3 different ultrafil- trate flow rates. Rezafungin adsorption to the CRRT appara- tus was determined for each hemodiafilter. Results: The sieving coefficient $\left(\mathrm{S}_{\mathrm{C}}\right)$ from $\mathrm{CVVH}$ with 3 different ultrafil- trate flow rates was 0 for both HF1400 and Multiflow-150 hemodiafilters, while urea $S_{C}$ was approximately 1 at all flow rates. Hemodiafilter type and ultrafiltrate flow rate did not}

\begin{tabular}{ll}
\hline KARGER & $\begin{array}{l}\text { (c) } 2018 \text { The Author(s) } \\
\text { Published by S. Karger AG, Basel }\end{array}$ \\
E-Mail karger@karger.com & This article is licensed under the Creative Commons Attribution- \\
www.karger.com/bpu & $\begin{array}{l}\text { NonCommercial-NoDerivatives 4.0 International License (CC BY- } \\
\text { NC-ND) (http://www.karger.com/Services/OpenAccessLicense). } \\
\text { Usage and distribution for commercial purposes as well as any dis- } \\
\text { tribution of modified material requires written permission. }\end{array}$
\end{tabular}

influence $\mathrm{CL}_{T M}$. Rezafungin adsorption to the CVVH apparatus was not observed for either hemodiafilter. Conclusion: Rezafungin is not removed by CVVH by membrane adsorption or via $\mathrm{CL}_{\mathrm{TM}}$. Ultrafiltrate flow rates and hemodiafilter types are unlikely to influence rezafungin $\mathrm{CL}_{\mathrm{TM}}$. No dosage adjustment of rezafungin is likely required for critically ill patients receiving $\mathrm{CVVH}$.

(c) 2018 The Author(s)
Published by S. Karger AG, Basel

\section{Introduction}

The incidence of acute kidney injury (AKI) in an intensive care setting is between 20 and 50\% [1]. Despite several technological advances in renal replacement therapies (RRT), the mortality rate remains in the range 50-60\% for patients with AKI requiring RRT [2]. Sepsis is the most common cause of death in critically ill pa- 
tients with AKI [3]. Systemic fungal infections represent a serious clinical complication in patients requiring RRT in the intensive care unit setting [4]. Some have suggested that inappropriate antibiotic dosing in these patients receiving continuous RRT (CRRT) is an important contributor to this high mortality rate $[5,6]$ and, similarly, inadequate antifungal dosing may lead to both treatment failure and resistance development [7, 8]. Echinocandins are considered the first-line antifungal therapy for invasive candidiasis, especially in critically ill patients [9].

Rezafungin is a novel long-acting echinocandin and its disposition during CRRT is unknown. Its high plasma protein binding ( $\sim 97-99 \%)$ suggests that rezafungin would be poorly removed by RRT [10]. However, there is a potential for some transmembrane clearance $\left(\mathrm{CL}_{\mathrm{TM}}\right)$ with contemporary hemodiafilters due to its relatively small volume of distribution $(35 \mathrm{~L})$ and molecular weight $(1,285 \mathrm{Da})$. CRRT has the ability to remove relatively large drugs (MW 1,000-2,000 Da) compared to hemodialysis, as CRRT operates 24-h/day using high-permeability hemodiafilters $[11,12]$. In addition, studies suggest that anidulafungin (with a similar chemical structure to rezafungin) binds to CRRT membranes [13]. The purpose of this ex vivo study is to quantify drug removal by $\mathrm{CL}_{\mathrm{TM}}$ and CRRT circuit adsorption. As rezafungin is administered once weekly and therapeutic drug monitoring is not available, it is essential to understand the CRRT influence on rezafungin pharmacokinetics to ensure sufficient rezafungin exposure.

\section{Materials and Methods}

This study assessed drug adsorption and $\mathrm{CL}_{\mathrm{TM}}$ using a continuous venovenous hemofiltration (CVVH) configuration. This validated ex vivo CVVH model [14-17] utilized $1 \mathrm{~L}$ of $\mathrm{pH}$ regulated, citrate-anti-coagulated bovine blood (Animal Technologies, Tyler, TX, USA). Using a Braun Diapact ${ }^{\mathrm{TM}}$ CRRT system (Braun, Bethlehem, PA, USA), 2 different hemodiafilters were tested. HF1400 (Polyarylethersulfone, Baxter Healthcare, Deerfield, IL, USA; surface area $1.4 \mathrm{~m}^{2}$ ) and the Multiflow-150 (AN69, Baxter Healthcare, Deerfield, IL, USA; surface area $1.5 \mathrm{~m}^{2}$ ) hemodiafilters were evaluated. New bovine blood, new hemodiafilters, and new CRRT tubing sets were used in each experiment. The blood was continuously stirred and heated to $37^{\circ} \mathrm{C}$ in a water bath during all experiments. Reconstituted rezafungin (Lot number AMU252277; Cidara Therapeutics, San Diego, CA, USA) was added to blood to achieve the final concentration of $\sim 30 \mathrm{mg} / \mathrm{L}$ (approximating the plasma peak concentration following multiple once-weekly $400 \mathrm{mg}$ doses [10]). Urea (Lot number 30K0221; Sigma, St. Louis, MO, USA) was used as a control and added to the blood to produce a blood urea nitrogen concentration of $\sim 75 \mathrm{mg} / \mathrm{dL}$.

\section{Adsorption and Degradation}

The purpose of the 1-h adsorption study was to determine if the drug adsorbs to the hemodiafilter or CRRT apparatus. Blood flow rates of $200 \mathrm{~mL} / \mathrm{min}$ with an ultrafiltration rate $\left(\mathrm{Q}_{\mathrm{uf}}\right)$ of $33 \mathrm{~mL} / \mathrm{min}$ were used. This allowed rezafungin to have maximal contact with the membrane, both on the blood side and via convection through the hemodiafilter membrane. A closed system was developed to maintain a constant volume in the extracorporeal system. Ultrafiltrate was used as the replacement fluid that was returned to the blood as a postfilter replacement fluid to maintain a constant blood volume. The CRRT machine was primed with normal saline before the operation; consequently, the urea and rezafungin dilution caused by the residual priming solution was accounted for. Blood samples were collected from the pre-filter port at 0 (baseline), 5 (at which time mixing was complete), 10, 20,30, and 60 min to assess adsorption over time. Previous studies have shown that membrane adsorption happens rapidly and is nearly complete in the first hour [18]. Each experiment was repeated 6 times with new hemodiafilters and tubing sets.

Degradation experiments $(n=6)$ were performed to ensure that the solutes were stable while placed in the $37^{\circ} \mathrm{C}$ blood for the 1 -h experiments. One liter of bovine blood was prepared identically as stated above. Urea was also added in this experiment acting as a control because it is known to be stable at $37^{\circ} \mathrm{C}$ for an hour and does not bind to hemodiafilters and to the CRRT apparatus [19]. Blood samples were collected from the flask at 0 (baseline), 5, $10,20,30$, and 60 min to detect degradation. Equations that were used to calculate dilution factor and adsorption are as follows:

$$
\% \text { Dilution factor }=\left(\frac{[\text { Ureat } 0]-[\text { Ureatx }]}{[\text { Ureat }]]}\right) \times 100
$$

where $\left[\mathrm{Urea}_{\mathrm{t} 0}\right]=$ urea concentration in the pre-filter at time 0

$\left[\mathrm{Urea}_{\mathrm{tx}}\right]=$ urea concentration in the pre-filter at time $\mathrm{x}$

$$
\% \text { Adsorption }=\left(\frac{\left[\mathrm{C}_{5}\right]-\left[\mathrm{C}_{60}\right]}{\left[\mathrm{C}_{5}\right]} \times 100\right)-\text { Dilution factor }- \text { Degradation }
$$

where $\left[\mathrm{C}_{5}\right]=$ concentration of pre-filter rezafungin at $5 \mathrm{~min}$

$$
\left[\mathrm{C}_{60}\right]=\text { concentration of pre-filter rezafungin at } 60 \mathrm{~min} \text {. }
$$

\section{Continuous Hemofiltration}

The procedures for the CVVH study were similar to those described in our previous ex vivo studies $[14,15]$. The $\mathrm{CL}_{\mathrm{TM}}$ of urea and rezafungin were evaluated with different blood flow rates, $\mathrm{Q}_{\mathrm{uf}}$ and hemodiafilters (Table 1). In a closed-loop system, the formed ultrafiltrate was returned to the blood as a post-filter replacement fluid downstream from the post-hemodiafilter blood sampling port. Figure 1 shows the schematic of the ex vivo post-filter replacement CVVH system. Pre- and post-hemodiafilter blood samples and ultrafiltrate samples were always collected concurrently. Samples were collected after 12 min when ultrafiltration rate was operated at $1 \mathrm{~L} / \mathrm{h}$. Then, ultrafiltration rate was changed to $2 \mathrm{~L} / \mathrm{h}$. Samples from these 3 ports were collected after 6 min when ultrafiltration rate was operated at $2 \mathrm{~L} / \mathrm{h}$. Lastly, ultrafiltration rate was changed to $3 \mathrm{~L} / \mathrm{h}$ and samples were collected after $4 \mathrm{~min}$. These sampling times were chosen to allow sufficient time for the ultrafiltrate to reflect the rezafungin concentrations arising from each of the different ultrafiltrate rates. Six experiments were conducted 
Table 1. $\mathrm{Q}_{\mathrm{b}}, \mathrm{Q}_{\mathrm{uf}}$, and types of hemodiafilters that were used for CVVH ex vivo study

\begin{tabular}{|c|c|c|c|c|c|}
\hline \multicolumn{3}{|c|}{ Hemodiafilter: HF1400 $(n=6)$} & \multicolumn{3}{|c|}{ Hemodiafilter: multiflow-150 $(n=6)$} \\
\hline $\mathrm{Q}_{\mathrm{b}}, \mathrm{mL} / \mathrm{min}$ & $\mathrm{Q}_{\mathrm{uf}}, \mathrm{mL} / \mathrm{min}$ & sampling time, $\min$ & $\mathrm{Q}_{\mathrm{b}}, \mathrm{mL} / \mathrm{min}$ & $\mathrm{Q}_{\mathrm{uf}}, \mathrm{mL} / \mathrm{min}$ & sampling time, min \\
\hline 200 & 16.7 & 12 & 200 & 16.7 & 12 \\
\hline 200 & 33.3 & 6 & 200 & 33.3 & 6 \\
\hline 400 & 50.0 & 4 & 400 & 50.0 & 4 \\
\hline
\end{tabular}

$\mathrm{Q}_{\mathrm{b}}$, blood flow rate; $\mathrm{Q}_{\mathrm{uf}}$, ultrafiltration rate.

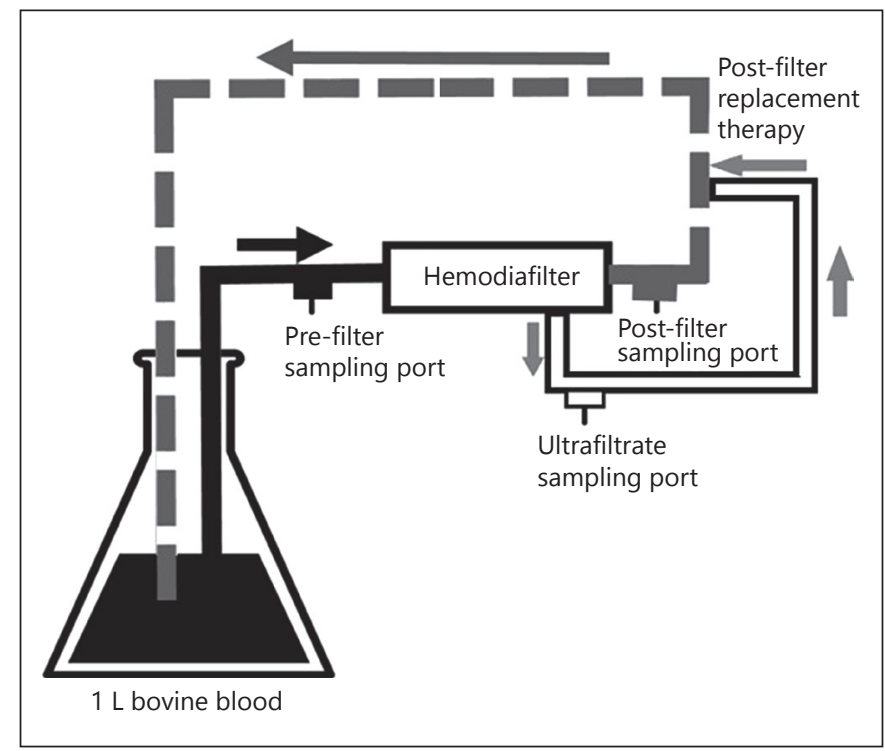

Fig. 1. Schematic representation of the ex vivo post-filter replacement CVVH system.

for each hemodiafilter, and new hemodiafilters and CRRT apparatus were used for each experiment. Sieving coefficient $\left(\mathrm{S}_{\mathrm{C}}\right)$ and $\mathrm{CL}_{\mathrm{TM}}$ for post-filter replacement were calculated as follows [15]:

$$
\mathrm{S}_{\mathrm{C}}=\frac{\mathrm{Cuf}_{\mathrm{uf}}}{\left(\mathrm{C}_{\mathrm{a}}+\mathrm{C}_{\mathrm{v}}\right) / 2}
$$

where $\mathrm{C}_{\mathrm{uf}}=$ concentration in the ultrafiltrate

$\mathrm{C}_{\mathrm{a}}=$ concentration of solute in the pre-filter

$\mathrm{C}_{\mathrm{v}}=$ concentration of solute in the post-filter

$$
\mathrm{CL}_{\mathrm{TM}}=\left(\mathrm{S}_{\mathrm{C}} \times \mathrm{Q}_{\mathrm{uf}}\right)
$$

where $S_{C}=$ observed sieving coefficient

$\mathrm{Q}_{\mathrm{uf}}=$ ultrafiltration rate.

\section{Sample Analysis}

All blood samples were centrifuged at 3,000 rpm for $10 \mathrm{~min}$. The plasma and ultrafiltrate samples were transferred to cryovials in duplicate. These samples were stored at $-80^{\circ} \mathrm{C}$ until analysis.
Blood urea nitrogen concentrations were analyzed with Advia 1800 (Siemens Healthcare Diagnostic Inc., Tarrytown, NY, USA) with lower limit of quantification of $5 \mathrm{mg} / \mathrm{dL}$. Rezafungin concentrations were measured by a Shimadzu HPLC coupled to an AB Sciex 5500 triple quadrupole mass spectrometer operating in selected reaction monitoring mode. The method employed triplicate standard calibrators prepared in ultrafiltrate using $d 9$ - rezafungin as internal standard and quantitation by analyte/internal standard area ratio versus concentration. For the degradation/adsorption samples, the calibration range was $1.00-50.0 \mu \mathrm{g} / \mathrm{mL}$ and the accuracy, as measured by the percent of nominal, was $84.3-141 \%$, and precision, as measured by percent coefficient of variation (\%CV), was $1.88-12.8 \%$. The analyses was quantified by a fit-for-purpose LC-MS/MS method aimed at determining if there was significant degradation or adsorption. The accuracy of $141 \%$ occurred at the lowest limit of quantitation, $1 \mu \mathrm{g} / \mathrm{mL}$, and was deemed acceptable for this degradation/adsorption sample analysis, since most samples quantified well above this concentration (average $25 \mu \mathrm{g} /$ $\mathrm{mL})$. For the CVVH samples, the calibration range encompassed $0.500-50.0 \mu \mathrm{g} / \mathrm{mL}$ and the accuracy was $70.8-118 \%$ with $\% \mathrm{CV}$ of $4.37-8.65 \%$.

\section{Data Analysis}

A power analysis calculation indicated that 6 experiments were required to detect a $25 \%$ of difference in the extent of rezafungin adsorption. Similarly, $6 \mathrm{CVVH}$ experiments with each hemodiafilter were required to detect a $25 \%$ difference in rezafungin $\mathrm{CL}_{\mathrm{TM}}$ between hemodiafilters (http://powerandsamplesize.com/Calculators/Compare-2-Means/2-Sample-Equality). Assumptions used in these calculations included a power of $90 \%$ and a standard deviation of $10 \%$ with a significance level of $p<0.05$. Two-tailed, unpaired $t$ test was used to compare differences between the 2 hemodiafilters, and analysis of variance was used to compare different $\mathrm{Q}_{\mathrm{uf}}$ within each hemodiafilter type.

\section{Results}

No rezafungin degradation was observed after $1 \mathrm{~h}$ in blood at $37^{\circ} \mathrm{C}$. The mean $\pm \mathrm{SD}$ of rezafungin concentrations $(\mu \mathrm{g} / \mathrm{mL})$ were $28.4 \pm 10$ (at $5 \mathrm{~min}$ ), $30.1 \pm 7$ (at $10 \mathrm{~min}$ ), $32.6 \pm 3$ (at $20 \mathrm{~min}$ ), $32.9 \pm 3$ (at $30 \mathrm{~min}$ ), and $34.5 \pm 2$ (at $60 \mathrm{~min})$. Neither rezafungin nor urea ad- 


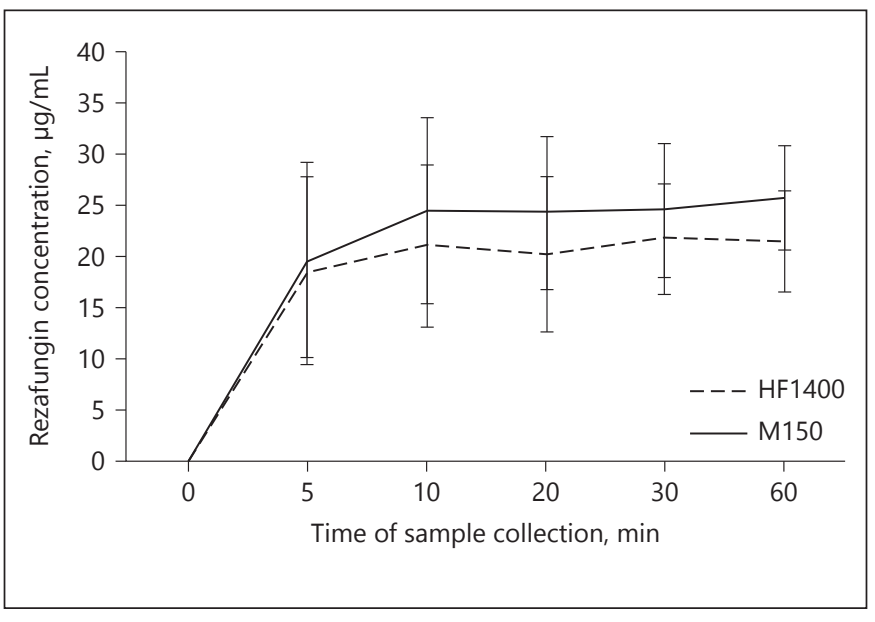

Fig. 2. Rezafungin concentration at different sampling times during the adsorption study (mean $\pm \mathrm{SD})$.

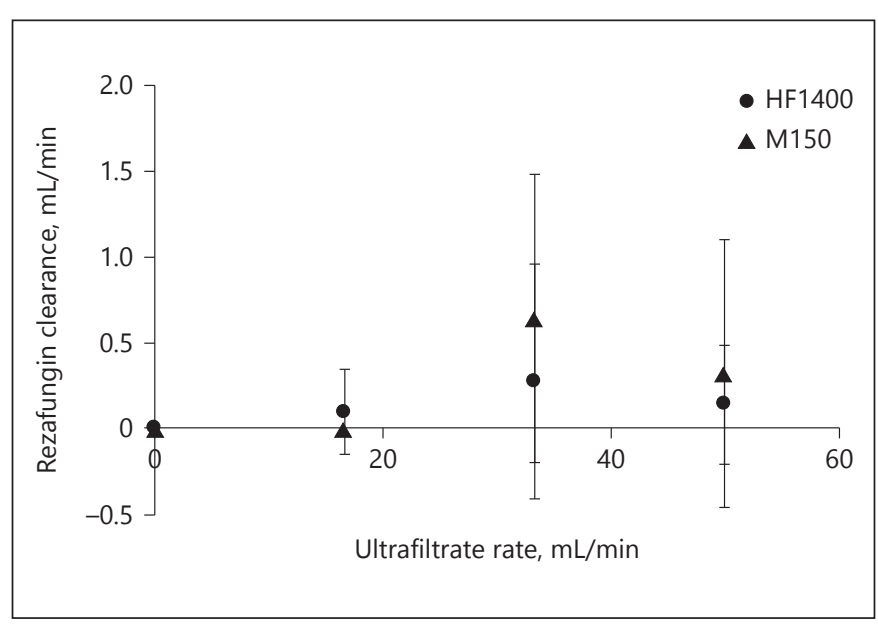

Fig. 3. Rezafungin transmembrane clearance during continuous hemofiltration (mean $\pm \mathrm{SD})$.

Table 2. Sieving coefficients of rezafungin and urea during CVVH experiments

\begin{tabular}{llllll}
\hline $\begin{array}{l}\text { Ultrafiltration } \\
\text { rate, } \mathrm{mL} / \mathrm{min}\end{array}$ & \multicolumn{2}{l}{$\mathrm{HF1400}(n=6$, mean $\pm \mathrm{SD})$} & & \multicolumn{2}{l}{ Multiflow-150 $(n=6$, mean $\pm \mathrm{SD})$} \\
\cline { 2 - 3 } & rezafungin & urea & & rezafungin & urea \\
\hline 36.67 & 0 & $1.0 \pm 0.1$ & & 0 & $1.0 \pm 0.1$ \\
50 & 0 & $1.0 \pm 0.1$ & & 0 & $1.0 \pm 0.1$ \\
\hline
\end{tabular}

Both hemodiafilter types and 3 ultrafiltration rates did not influence the rezafungin clearance during CVVH experiments.

sorption was observed with either hemodiafilter type (Fig. 2). Hemodiafilter types (HF1400 $p=0.5$; Multiflow-150 $p=0.2$ ) and ultrafiltration rates did not influence the adsorption of rezafungin. As illustrated in Table 2 , urea $S_{C}$ consistently approximated 1 and urea $\mathrm{CL}_{\mathrm{TM}}$ was dependent on ultrafiltrate production rate. Rezafungin $\mathrm{S}_{\mathrm{C}}$ values were zero with 3 different $\mathrm{Q}_{\mathrm{uf}}$ in both hemodiafilter types (Table 2). This ex vivo study indicates rezafungin is not cleared by $\mathrm{CVVH}$ either by $\mathrm{CL}_{\mathrm{TM}}$ (Fig. 3) or by adsorption. The change in $\mathrm{Q}_{\mathrm{uf}}$ and types of hemodiafilter did not influence the $\mathrm{CL}_{\mathrm{TM}}(p>0.05)$.

\section{Discussion}

No dosage adjustment for CRRT is necessary for currently marketed echinocandins (caspofungin, micafungin, anidulafungin) due to high protein binding and predominant non-renal clearance. For instance, caspofungin's $\mathrm{CL}_{\mathrm{TM}}$ by $\mathrm{CVVH}$ has been reported to be approximately $8 \mathrm{~mL} / \mathrm{h}$ (1.8\% of total clearance) and $5.9 \mathrm{~mL} / \mathrm{h}$ (2.5\% of total clearance) in continuous venovenous hemodialysis (CVVHD) [20]. A more recent study of caspofungin clearance by CVVH and continuous venovenous hemodiafiltration (CVVHDF) found a similarly negligible clearance by these therapies ( 48 and $42 \mathrm{~mL} / \mathrm{h}$, respectively) [21]. Likewise, micafungin CVVHDF clearance using the same AN69 membrane employed in our study was negligible in a clinical study of 10 patients [22]. In contrast, anidulafungin has conflicting data regarding adsorption. One report states that the influence of CRRT on anidulafungin elimination is negligible, since there was no anidulafungin adsorption to synthetic surfaces, and ultradiafiltrate concentrations were below the assay limit of detection [23]. However, probable anidulafungin binding to CRRT membranes was observed in a clinical trial $(n=10)$ when pre- and 
post-filter anidulafungin concentrations were compared, even though no drug was detected in the ultrafiltrate [13]. Our study findings did not detect rezafungin hemodiafilter binding, and found no measurable drug $\mathrm{CL}_{\mathrm{TM}}$.

Limitations of this study include the fact that we used bovine blood as the study matrix. Although bovine albumin occurs in approximately the same concentrations $(\sim 3 \mathrm{~g} / \mathrm{dL})$ as human serum albumin in critically ill patients receiving CRRT, bovine albumin is different than human albumin and protein binding may differ. However, comparisons of previous ex vivo studies using the same methods to in vivo CRRT trials of the same drug $[16,17]$ have shown good agreement in clearance estimates. Our findings of an SC not different from zero in the ex vivo model are very consistent with what is known about the extensive (97-99\%) protein binding in humans receiving rezafungin [10].

\section{Conclusion}

Rezafungin is a novel, long-acting echinocandin, which is likely to be used in critically ill patients receiving CRRT. Based on the $S_{C}$ observed in this ex vivo study, rezafungin is unlikely to be adsorbed nor cleared by any form of CRRT. Rezafungin is administered once weekly and dosage adjustment is not likely to be required for critically ill patients receiving CRRT.

\section{Disclosure Statement} Inc.

Dr. Mueller received grant funding from Cidara Therapeutics,

\section{Funding Source}

This investigator-initiated study was funded by Cidara Therapeutics, Inc.

\section{References}

1 Case J, Khan S, Khalid R, Khan A: Epidemiology of acute kidney injury in the intensive care unit. Crit Care Res Pract 2013;2013: 479730.

-2 Barbar SD, Binquet C, Monchi M, Bruyere R, Quenot JP: Impact on mortality of the timing of renal replacement therapy in patients with severe acute kidney injury in septic shock: the IDEAL-ICU study (initiation of dialysis early versus delayed in the intensive care unit): study protocol for a randomized controlled trial. Trials 2014;15: 270.

- 3 Woodrow G, Turney JH: Cause of death in acute renal failure. Nephrol Dial Transplant 1992;7:230-234.

-4 Leroy O, Gangneux JP, Montravers P, Mira JP, Gouin F, Sollet JP, Carlet J, Reynes J, Rosenheim M, Regnier B, Lortholary O; AmarCand Study Group: Epidemiology, management, and risk factors for death of invasive Candida infections in critical care: a multicenter, prospective, observational study in France (2005-2006). Crit Care Med 2009;37: 1612-1618.

5 Kielstein JT, David S: Pro: Renal replacement trauma or Paracelsus 2.0. Nephrol Dial Transplant 2013;28:2728-2731;discussion 27312723.

-6 Lewis SJ, Mueller BA: Antibiotic dosing in critically ill patients receiving CRRT: underdosing is overprevalent. Semin Dial 2014;27 441-445.

7 Sinnollareddy M, Peake SL, Roberts MS, Lipman J, Roberts JA: Using pharmacokinetics and pharmacodynamics to optimise dosing of antifungal agents in critically ill patients: a systematic review. Int J Antimicrob Agents 2012;39:1-10.

-8 Sinnollareddy MG, Roberts JA, Lipman J, Akova M, Bassetti M, De Waele JJ, Kaukonen KM, Koulenti D, Martin C, Montravers P, Rello J, Rhodes A, Starr T, Wallis SC, Dimopoulos G: Pharmacokinetic variability and exposures of fluconazole, anidulafungin, and caspofungin in intensive care unit patients: data from multinational defining antibiotic levels in intensive care unit (DALI) patients Study. Crit Care 2015; 19:33

$\checkmark 9$ Pappas PG, Kauffman CA, Andes DR, Clancy CJ, Marr KA, Ostrosky-Zeichner L, Reboli AC, Schuster MG, Vazquez JA, Walsh TJ, Zaoutis TE, Sobel JD: Clinical practice guideline for the management of Candidiasis: 2016 Update by the Infectious Diseases Society of America. Clin Infect Dis 2016;62:e1e50.

10 Sandison T, Ong V, Lee J, Thye D: Safety and pharmacokinetics of CD101 IV, a novel echinocandin, in healthy adults. Antimicrob Agents Chemother 2017;61:pii:e01627e01716.

-11 Chaijamorn W, Jitsurong A, Wiwattanawongsa $\mathrm{K}$, Wanakamanee $\mathrm{U}$, Dandecha $\mathrm{P}$ : Vancomycin clearance during continuous venovenous haemofiltration in critically ill patients. Int J Antimicrob Agents 2011;38:152156.

12 Eyler RF, Vilay AM, Nader AM, Heung M, Pleva M, Sowinski KM, DePestel DD, Sorgel F, Kinzig M, Mueller BA: Pharmacokinetics of ertapenem in critically ill patients receiving continuous venovenous hemodialysis or hemodiafiltration. Antimicrob Agents Chemother 2014;58:1320-1326.

13 Leitner JM, Meyer B, Fuhrmann V, Saria K, Zuba C, Jager W, Bohmdorfer M, Thalhammer F: Multiple-dose pharmacokinetics of anidulafungin during continuous venovenous haemofiltration. J Antimicrob Chemother 2011;66:880-884.

14 Lewis SJ, Switaj LA, Mueller BA: Tedizolid adsorption and transmembrane clearance during in vitro continuous renal replacement therapy. Blood Purif 2015;40:6671.

15 Chaijamorn W, Shaw AR, Lewis SJ, Mueller BA: Ex vivo ceftolozane/tazobactam clearance during continuous renal replacement therapy. Blood Purif 2017;44:16-23.

-16 Churchwell MD, Pasko DA, Mueller BA Daptomycin clearance during modeled continuous renal replacement therapy. Blood $\mathrm{Pu}$ rif 2006;24:548-554.

17 Vilay AM, Grio M, Depestel DD, Sowinski KM, Gao L, Heung M, Salama NN, Mueller BA: Daptomycin pharmacokinetics in critically ill patients receiving continuous venovenous hemodialysis. Crit Care Med 2011;39: 19-25.

18 Clark WR, Macias WL, Molitoris BA, Wang $\mathrm{NH}$ : Plasma protein adsorption to highly permeable hemodialysis membranes. Kidney Int 1995;48:481-488.

19 Marjani A: Effect of storage time and temperature on some serum analytes. Internet J Lab Med 2006;2:2. 


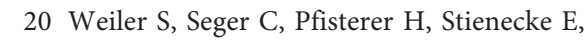
Stippler F, Welte R, Joannidis M, Griesmacher A, Bellmann R: Pharmacokinetics of caspofungin in critically ill patients on continuous renal replacement therapy. Antimicrob Agents Chemother 2013;57:40534057.

21 Roger C, Wallis SC, Muller L, Saissi G, Lipman J, Bruggemann RJ, Lefrant JY, Roberts JA: Caspofungin population pharmacokinet- ics in critically ill patients undergoing continuous veno-venous haemofiltration or haemodiafiltration. Clin Pharmacokinet 2017;56: 1057-1068.

22 Vossen MG, Knafl D, Haidinger M, Lemmerer R, Unger M, Pferschy S, Lamm W, Maier-Salamon A, Jager W, Thalhammer F: Micafungin plasma levels are not affected by continuous renal replacement therapy: experience in critically ill patients. Antimi- crob Agents Chemother 2017;61:pii:e02425e02516.

23 Aguilar G, Azanza JR, Carbonell JA, Ferrando C, Badenes R, Parra MA, Sadaba B, Navarro D, Puig J, Minana A, Garcia-Marquez C, Gencheva G, Gutierrez A, Marti FJ, Belda FJ: Anidulafungin dosing in critically ill patients with continuous venovenous haemodiafiltration. J Antimicrob Chemother 2014;69:16201623. 